\title{
Physical modelling of cave breakthrough into an overlying cave
}

\author{
D Cumming-Potvin Australian Centre for Geomechanics, University of Western Australia, \\ Australia
}

J Wesseloo Australian Centre for Geomechanics, University of Western Australia, Australia

SW Jacobsz University of Pretoria, South Africa

Y Potvin Australian Centre for Geomechanics, University of Western Australia, Australia

A Mooney Oz Minerals, Australia

\begin{abstract}
Access to the caving column in caving mines is limited and, as a result, the amount of data that can be collected (in particular observational data) is very limited. These limitations have resulted in a situation where the mechanisms of cave propagation and breakthrough are poorly understood. Cave breakthrough is of particular importance, as it is a crucial, high-risk stage of cave development. This is exacerbated by the increasingly high cave columns being mined in recent years. Breakthrough of a cave into an existing, overlying cave adds additional challenges as the connection at depth creates high induced stresses and large scale stress redistribution, sometimes resulting in large seismic events. Since visual access to the cave column is possible with physical modelling, it can deliver important insight into the caving mechanisms evolved. This is even more critical for situations of cave breakthrough into existing caves, as they are relatively rare in industry - limiting the ability to study them. In order to better understand these cave breakthrough events, a research project sponsored by Oz Minerals was created to model such situations in a geotechnical centrifuge. Four tests have been completed to date, the preliminary results of which show a potential mechanism of cave connection which has been previously unrecognised. This involves failure of the corners around the existing overlying cave when the cave back of the propagating cave below has only reached one-half to three-quarters of the vertical height of the cave column. The analysis is only in the early stages and further work is necessary to confirm the validity of the observed mechanism and rule out the possibility that it is an artefact resulting from the test methodology. Further research will focus on more detailed analysis of the results and further tests to better understand the observations from the first four tests.
\end{abstract}

\section{Introduction}

The propagation and breakthrough of caves, particularly into existing caves, is a complicated and poorly understood process, which is governed by a range of factors. There are a number of monitoring techniques which mines employ to try to track cave progression, however these are either indirect methods (seismic monitoring) or simple point measurements which are often discontinuous in time (TDR, open hole dipping, extensometers, smart markers etc.). Access to the caving column is inherently limited in caving mines. It is therefore difficult to install a comprehensive instrument programme and direct visual observations are only possible via a limited number of boreholes for a limited period of time. This results in a situation where the propagation of the cave is inferred, rather than known and the true nature of caving propagation is unknown. There is often even less opportunity to monitor cave breakthrough, as the danger posed by the breakthrough often limits staff access above the cave - further restricting the ability to make these readings/observations. 


\section{Uncertainty in the behaviour of the caving rock mass during breakthrough}

During breakthrough, areas above a cave experiences not only subsidence, but a period of significant stress re-adjustment which may induce increased seismicity and large seismic events, as seen in case studies at Northparkes, Palabora, Ridgeway and DOZ mines (Glazer 2018; Potvin \& Hudyma 2008; Srikant et al. 2007). This may be exacerbated in cases where the breakthrough is into an overlying cave, particularly at depth. The large seismic events often generated present a significant seismic risk to underground workers and infrastructure. It is of particular importance to understand the mechanisms involved, as cave breakthrough is an important, high-risk stage in any caving operation and will become increasingly common as cave mining continues to increasing depths and block heights in the future (Figure 1). The potential for rapid cave propagation during the breakthrough period can present a significant risk to resource recovery.

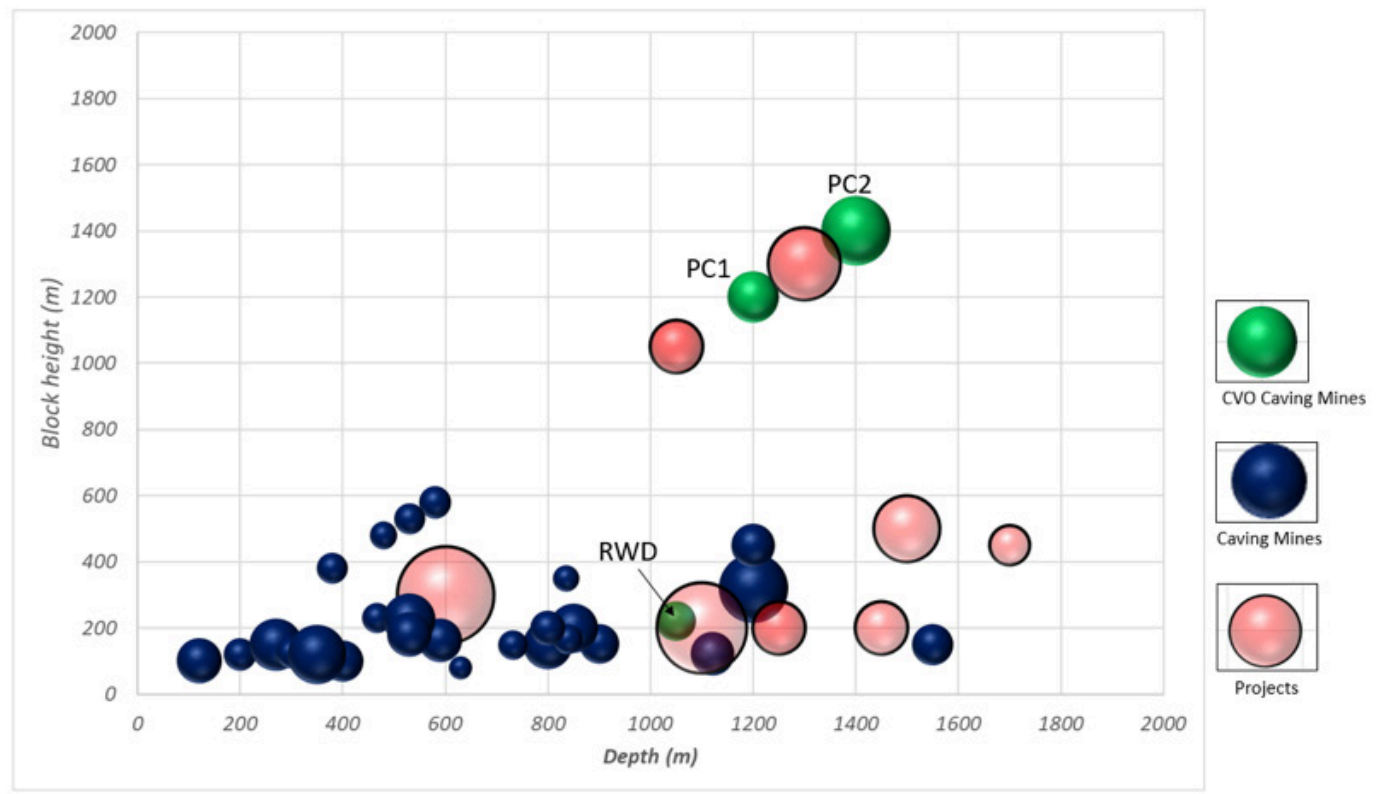

Figure 1 Compilation of existing and caving mines and projects showing the depth, block height and production (proportional to makers' size). After Cuello \& Newcombe (2018)

Despite its importance, the mechanisms involved in the process of cave breakthrough into existing caves are poorly understood and could differ significantly from those of cave propagation into virgin ground. This is in part due to lack of data and observations - there are few cave mines in the world who have experienced breakthrough into an overlying cave, and the process typically only lasts a few weeks to a few months. This, coupled with the inherent difficulties in observations of caving, result in very little industry knowledge of the mechanisms by which a cave breaks through into an existing cave. This challenge can start to be addressed through the use of physical models.

The use of the physical centrifuge modelling enables the reproduction of a caving process in a controlled laboratory environment with freedom of observation and instrumentation, which are not available in the field. It should be noted that the experiments detailed in this paper do not attempt to replicate a specific cave, but in this case, a generic breakthrough.

Recent work (Cumming-Potvin 2018; Cumming-Potvin et al. 2016a; b; Cumming-Potvin et al. 2018) extended the generally accepted Duplancic conceptual model of the caving procession (Duplancic \& Brady 1999) to incorporate a discontinuous fracture banding process not previously recognised. This work has shown that the mechanics of cave propagation is more complex than previously understood, and that further work is necessary to define the boundaries of, and governing factors driving different cave propagation mechanisms (Figure 2). 


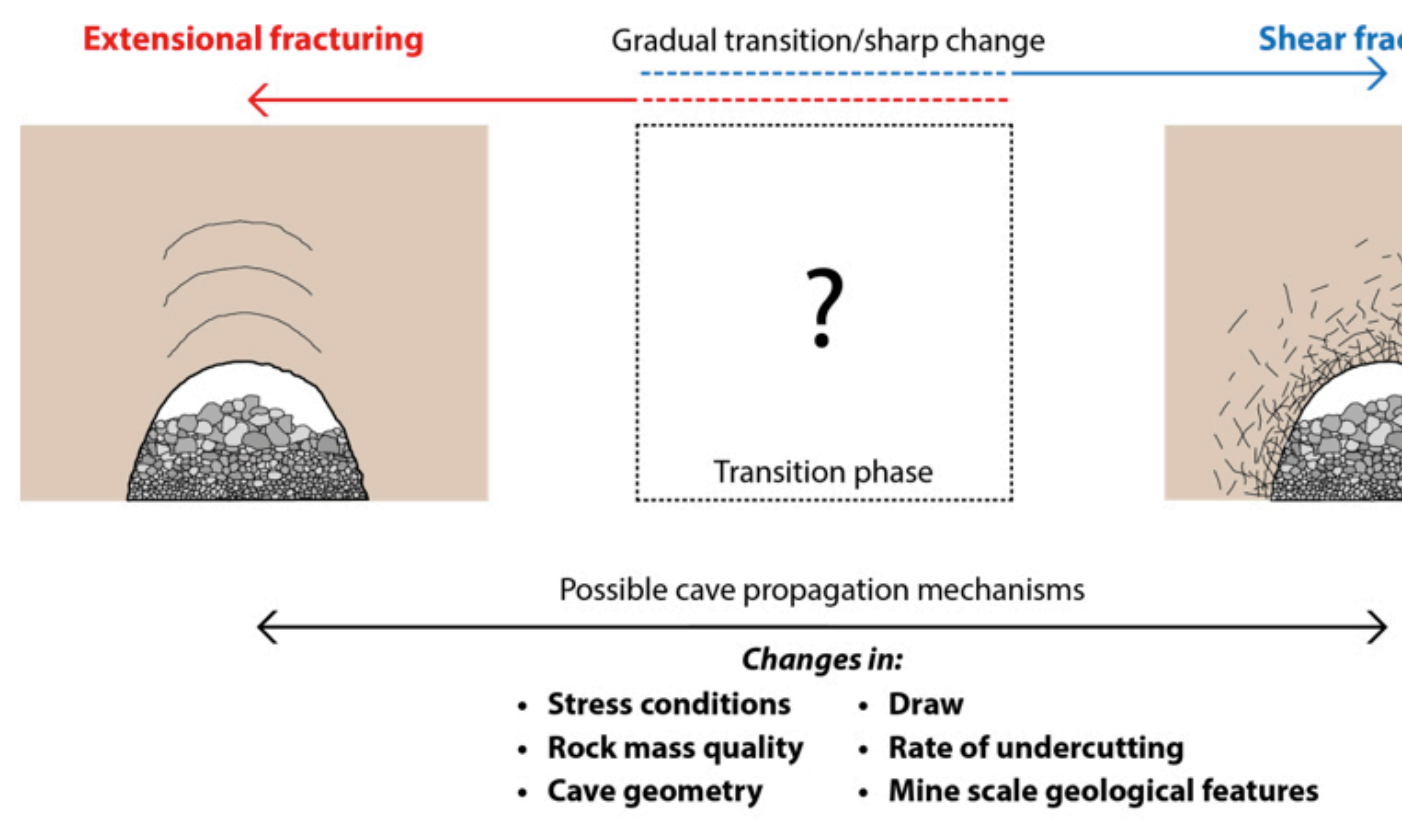

Figure 2 Speculative conceptual model of caving (Cumming-Potvin 2018)

To the author's knowledge, no previous physical models have been created which aim to simulate the breakthrough of a cave into an existing overlying cave, a situation that will become increasingly common in future deep multi-lift block caves and sub-level to block cave transitions. A research project, sponsored by Oz Minerals, was undertaken in order to bridge this gap.

\section{$3 \quad$ Experiment design}

When considering the design a physical model, a key consideration is the body forces of the sample material. If the body forces are not sufficiently replicated, the failure mechanism cannot be captured correctly (Wesseloo et al. 2020). Additionally, in cases where the modelled excavation is large in comparison to the depth below surface (such as caving), using externally applied forces is not a valid simplification of the stress field (Hoek 1965). Due to both of these factors, centrifuge loading was a requirement in the testing of these physical models. The University of Pretoria geotechnical centrifuge facility was used for this project. The facility includes a $150 \mathrm{~g}$-ton centrifuge with a $3 \mathrm{~m}$ radius and a range of on board instrumentation. A picture of the centrifuge is given in Figure 3 and more details on this facility can be found in Jacobsz et al. (2014).

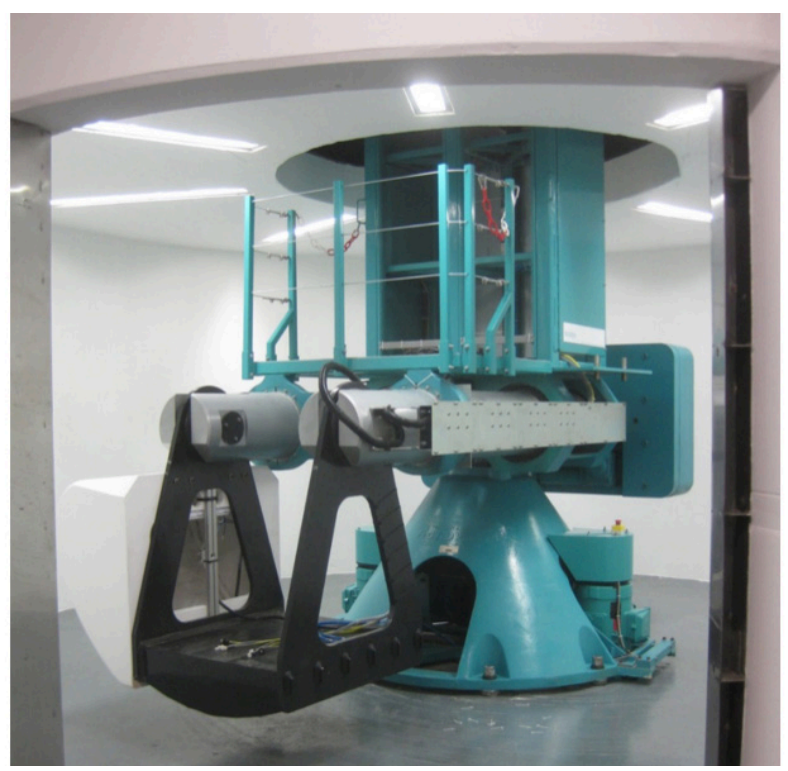

Figure 3 University of Pretoria geotechnical centrifuge 
The test followed the procedure outlined in Cumming-Potvin (2018), with adjustments to investigate cave breakthrough into a mature overlying cave. A sample (made from a clay/fly-ash mixture) was placed into a specially built frame for testing. The sample dimensions were $450 \mathrm{~mm}$ by $240 \mathrm{~mm}$ by $47 \mathrm{~mm}$, creating a quasi-two dimensional test. This was required in order to enable visual observations of the caving process. Underneath the sample were 5 electric linear actuators which could be controlled remotely. The front of the testing frame consisted of a $30 \mathrm{~mm}$ thick laminated toughened glass pane, which allowed visual observation of the tests. The sides of the sample were constrained by bladders filled with water, to control horizontal stress acting on the sample. The vertical stress in the sample was provided by the self-weight of the sample, multiplied by the acceleration of the centrifuge. A portion at the top of the sample was left as void, which was filled with particulate material to simulate an overlying cave. In two of the tests, the particulate material was sand and in the other two it was gravel. The sand was layered with different colours to allow visual tracking of caved material movement. At this stage no analysis has been completed on the impact of modifying the upper-cave material. The area next to the simulated existing cave material was filled with wooden spacers surcharged with brass weights. These were used due to the fragility of the sample material - creating a void large enough for testing in a sample would have resulted in a sample too fragile to handle. The wooden spacers allowed a sample with a smaller void to be used, while still providing confinement to the sample and simulated existing cave material. The entire frame was accelerated to $80 \mathrm{~g}$ in the centrifuge and the actuators below the sample were then retracted to allow a cave to propagate from the bottom of the sample until it broke through to the simulated overlying cave material above. A schematic of the tests is given in Figure 4.

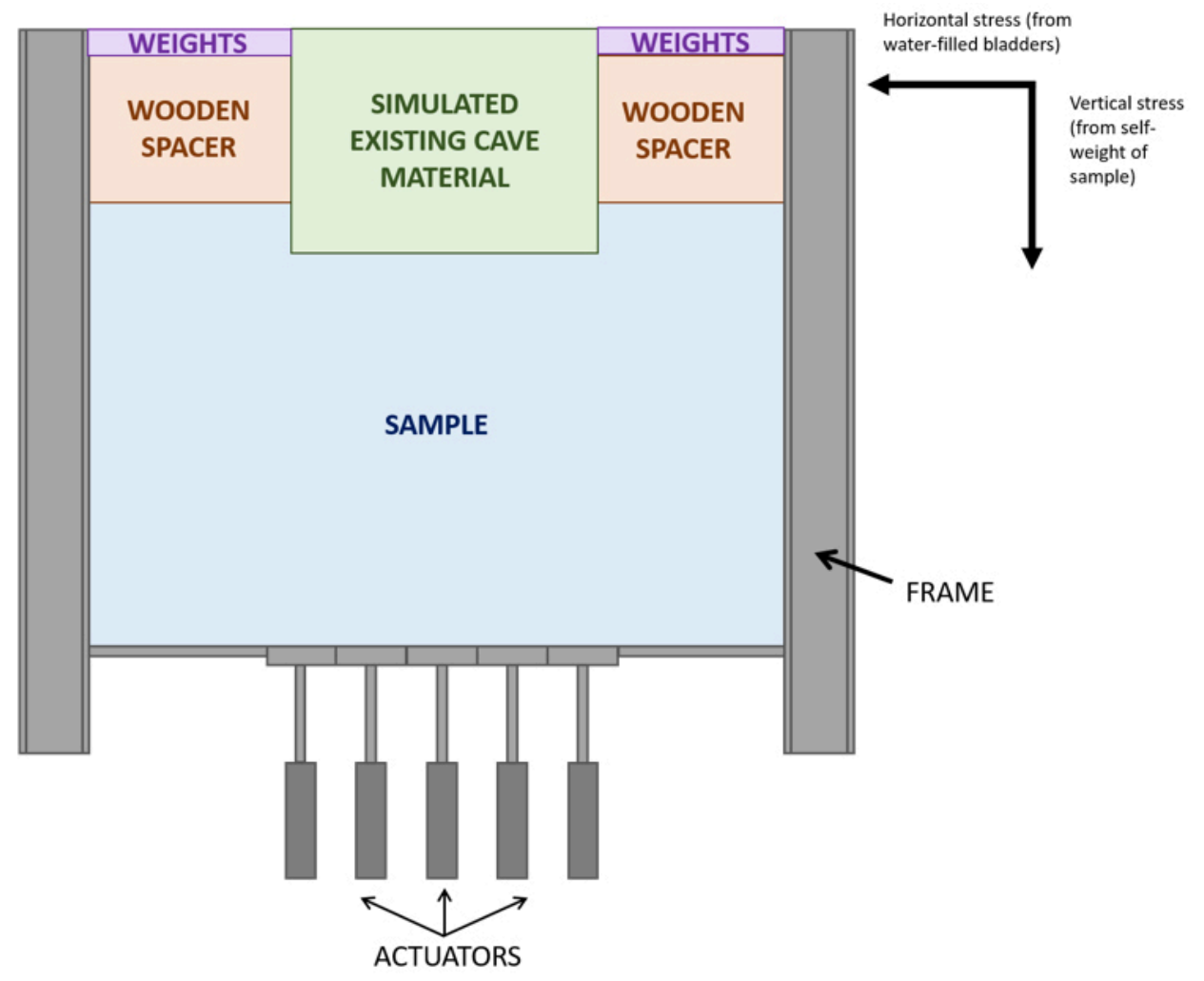

\section{Figure 4 Test schematic (not to scale)}

The sample material used was adjusted from that given in Cumming-Potvin (2018), a mixture of sand, cement, fly ash and water. The adjustments were to replace the sand and cement in the mix with kaolinite, and to adjust the relative proportions of the constituents. These changes were made in order to make the material more closely resemble rock in terms of brittleness. This is particularly important when considering the acoustic emission results, which rely on brittle failure to create strong enough acoustic signals to record. The scaling law governing similitude for a scale model such as this is given by: 


$$
\begin{gathered}
\frac{2 \cdot \tau_{m}}{l_{m} \cdot \rho_{m} \cdot g_{m}}=\frac{2 \cdot \tau}{l \cdot \rho \cdot g} \\
\frac{2 \cdot\left(f_{\tau} \cdot \tau\right)}{\left(f_{l} \cdot l\right) \cdot\left(f_{\rho} \cdot \rho\right)\left(f_{g} \cdot g\right)}=\frac{2 \cdot \tau}{l \cdot \rho \cdot g}
\end{gathered}
$$

Where:

$\tau_{m}, \tau=$ The shear stress for the model and the modelled scenario respectively

$\rho_{\mathrm{m},} \rho=$ The model and modelled material density

$I_{m} \mid=$ the model and modelled scale

$\mathrm{g}_{\mathrm{m}}=$ gravitational acceleration in the model

g $=$ gravitational constant $\approx 9.8 \mathrm{~m} / \mathrm{s}^{2}$

$f_{l}, f_{\rho}, f_{g}, f_{\tau} \quad$ scaling factors for the different parameters

Given the constraints on the model scale and model gravitational acceleration achievable in the centrifuge, the use of a sample material as strong as rock was not possible and thus the material strength needed to be scaled down to approximately $500 \mathrm{kPa}$. A more detailed discussion of this is given in Wesseloo et al. (2020). The breakdown of the sample material components by mass are listed in Table 1.

Table 1 Sample material breakdown (prior to curing)

\begin{tabular}{|c|c|}
\hline Material & \% (by mass) \\
\hline Kaolinite & 16.40 \\
\hline Fly Ash & 53.24 \\
\hline Water & 30.36 \\
\hline
\end{tabular}

The tests were well instrumented in order to extract the maximum possible value from each test. There are three on board cameras; a simple web camera for quick visual results, a Basler acA2000-165uc with an $8.5 \mathrm{~mm}$ F/1.3-16 lens and a Canon EOS 100D with a $40 \mathrm{~mm}$ fixed lens. These cameras (in particular the high speed Basler) will allow detailed investigation into the mechanism of failure around breakthrough, as the formation and coalescence of fractures can be observed. The displacement of the actuators is digitally recorded by the on-board acquisition system. An array of 6 Acoustic Emission (AE) sensors were also placed on the sides of the samples, to allow analysis of the acoustic signature of the breakthrough (analogous to microseismic monitoring in caving mines).

To date, four tests have been completed as part of the research project, however the analysis of the data is only at a preliminary stage at the time of writing this paper. More tests are also planned.

\section{$4 \quad$ Preliminary results}

In all four tests, the cave propagated via fracture banding - where a series of fractures parallel to the cave surface form ahead of the cave and eventually become the next cave surface (Cumming-Potvin et al. 2016a). An example of cave propagation through fracture banding from one of the tests is given in Figure 5. 

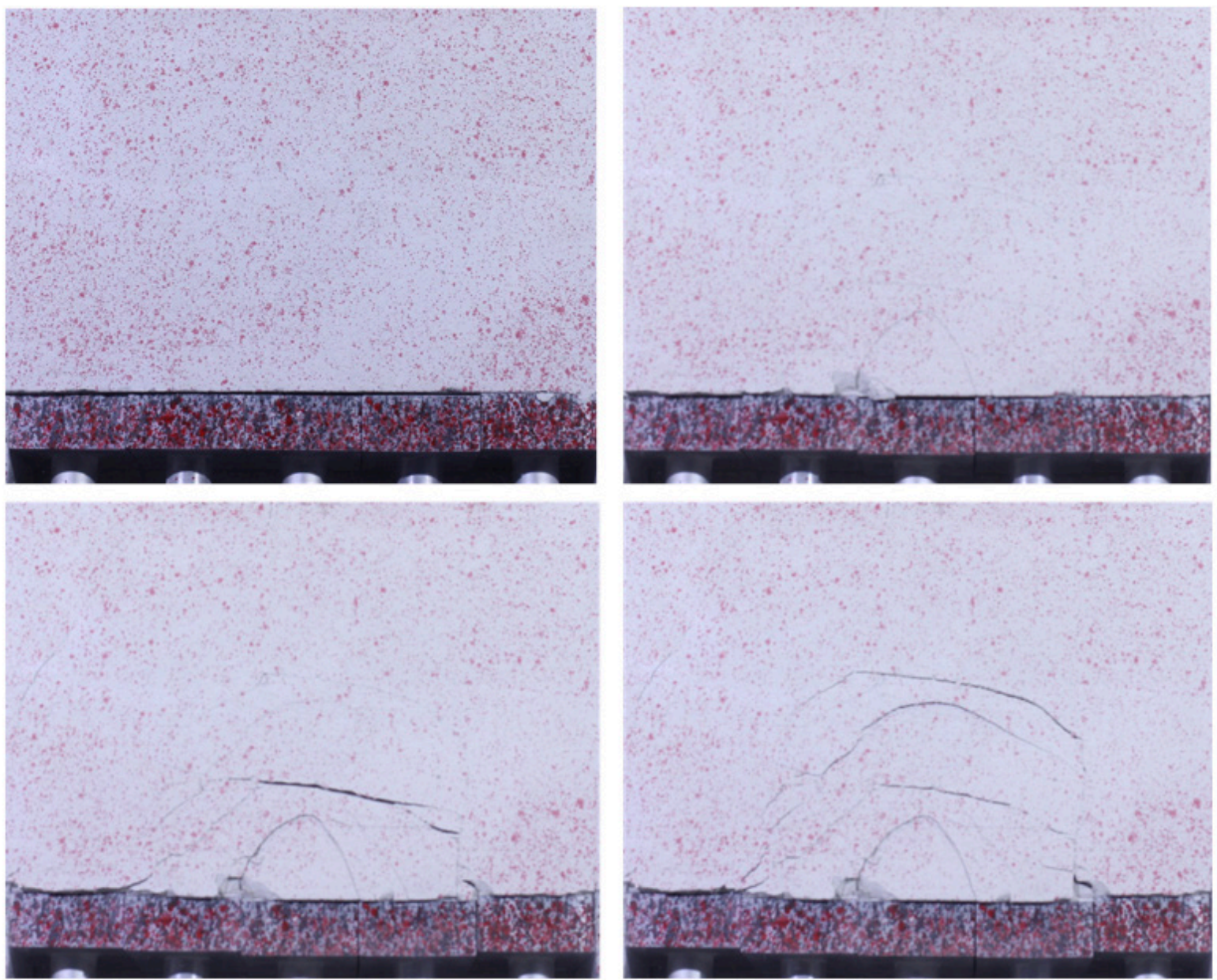

Figure 5 Example cave propagation through fracture banding

Preliminary analysis of the results of the tests indicate the possibility of a previously unrecognised mechanism of cave connection. In all four tests, the lower cave propagated to a height approximately one half to three-quarters of the distance between the actuators and the existing (upper) cave, before the breakthrough suddenly took place.

The application of the Duplancic model of caving (Duplancic \& Brady 1999) would suggest that the cave should gradually continue to propagate vertically and for the two caves to connect near the centre of the crown pillar. The connection in the centrifuge model, however, occurred rapidly at the corners of the upper cave, extending downward outside and sub-parallel to the lower caved zone. There is no significant further vertical propagation of the lower cave during the connection period. Examples from all four tests are given in Figure 6, with the lower cave back highlighted in green and the cave connection zones "connection cracks" highlighted in red, located outside the column. 

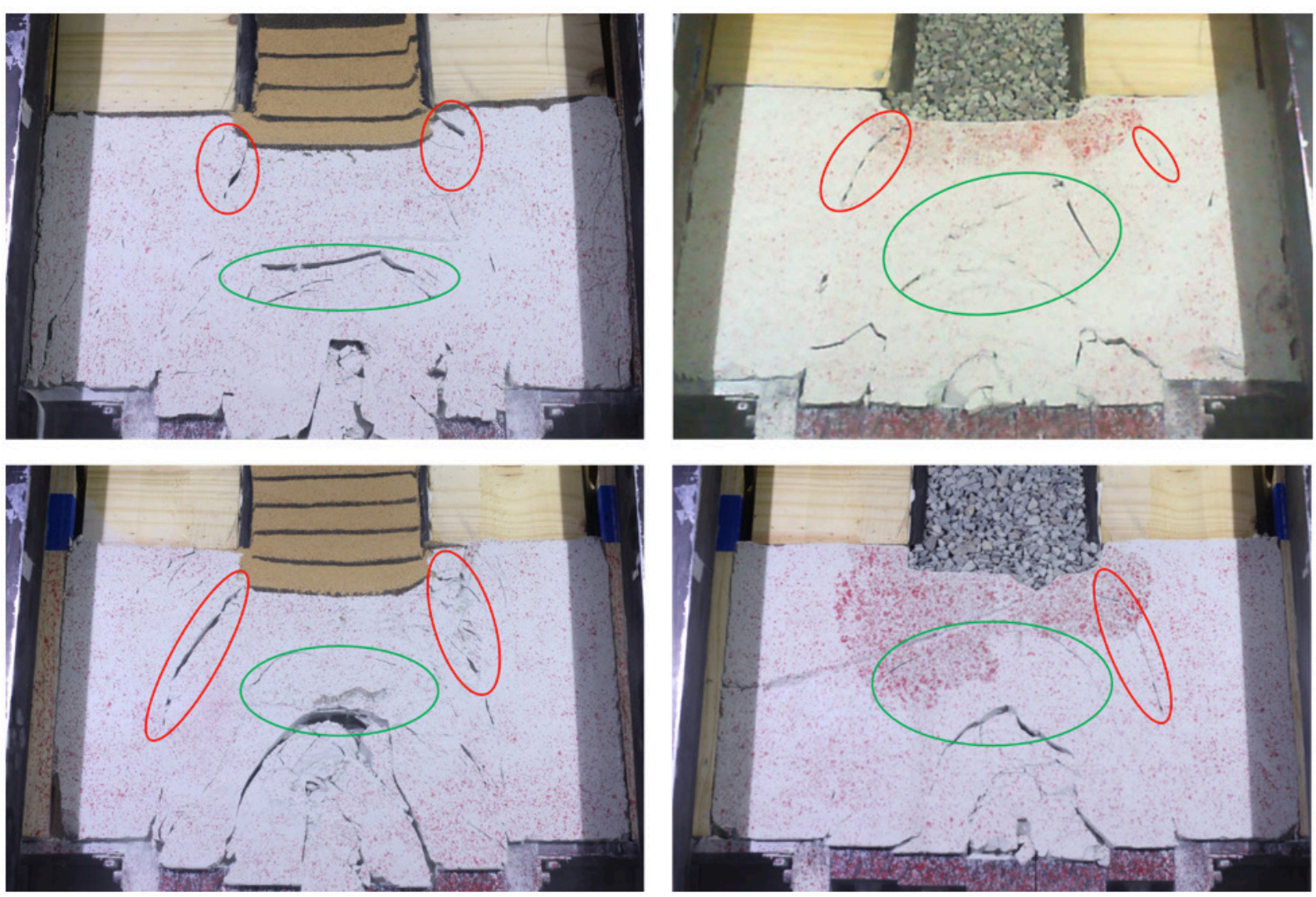

Figure 6 Example of observed mechanism of cave connection from each test. Cave connection failure zones circled in red, cave back of propagating (lower) cave circled in green

After these failure zones develop, the cave fully connect via a rapid, 'plug' style failure of the zone between the lower and upper caves. Confidence in the mechanisms observed at this stage of the test is limited, as the boundary conditions of the test are compromised.

Some interesting parallels can be drawn between these observations and the connections of Northparkes E 26 Lift 2 and Lift 1. Hudyma et al. (2008) studied the seismicity associated with the E-26 connection. They observed that the first $150 \mathrm{~m}$ cave propagation occurred relatively progressively, over a period of approximately 10 months (February 2003 - November 2004). However, the last $100 \mathrm{~m}$ of the crown separating the two lifts caved very rapidly during a period of one and a half months (November December 2004) (Figure 7). This behaviour is akin to the centrifuge cave progressing "normally" up to half to three quarters of the distance between the actuators and the upper cave, followed by a sudden failure of the crown separating the two caves, involving the formation of major cracks (highlighted in red in Figure 6).

Over 20 large events (local magnitude $\geq 1.0$ ) were generated during a two-week period (November 3 -16), as shown with yellow and orange markers, in the Magnitude-Time graph in Figure 8. Despite the large events, the overall event rate during this period was very low. The large events were generally not located in the crown pillar under Lift 1, but outside the column towards the upper part of Lift 2 (Figure 9). 


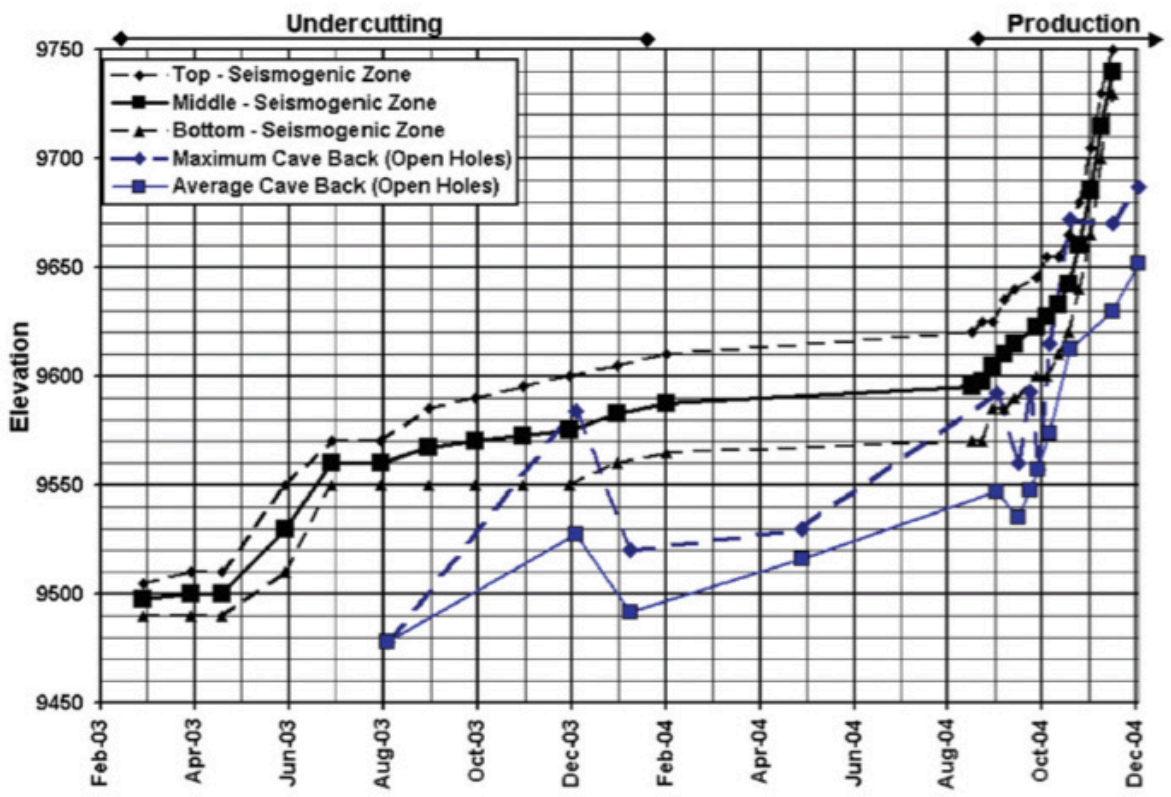

Figure 7 Elevation of the cave back measured by open holes (blue line and markers) along with estimated seismogenic zone (black line and markers). (Hudyma et al. 2007b)

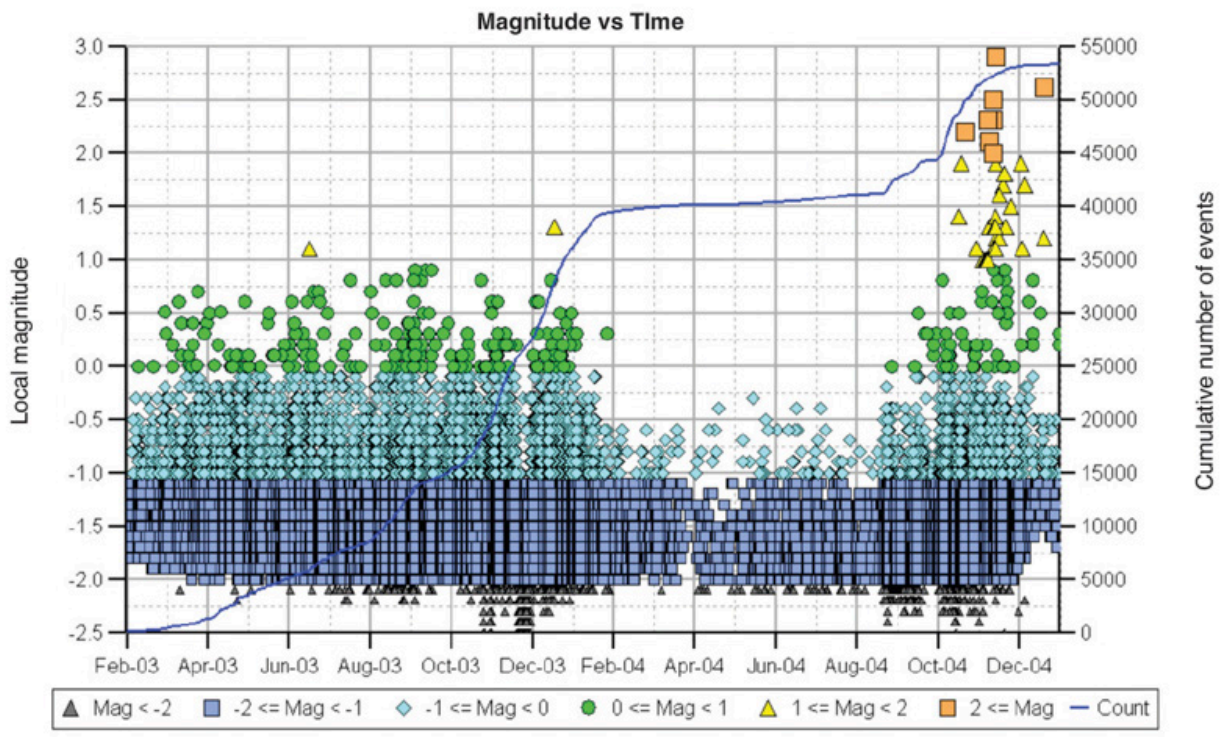

Figure 8 Magnitude-Time chart of seismicity at Northparkes E26 Lift 2. Note the increase in large magnitude events in late 2004 (Hudyma et al. 2007a)
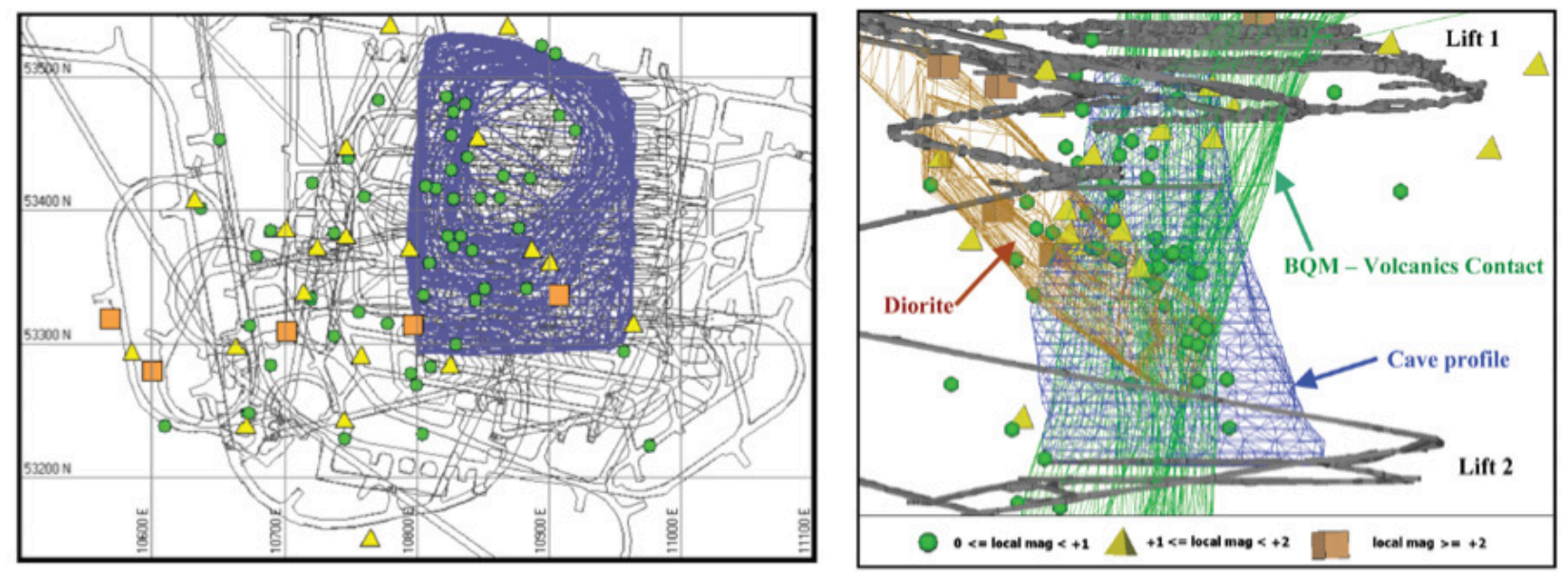

Figure 9 Location of the large events outside the column during the connection between Lift 2 and Lift 1 at Northparkes, plan view (left) and looking North-East (right) (Hudyma et al. 2007b) 
Very similar observations were made by Glazer \& Hepworth $(2005 ; 2006)$ where the last $100 \mathrm{~m}$ of crown pillar separating the open pit and the cave produced very little seismicity (within the column), but generated 12 large events (ML > 0.5). In both Northparkes and Palabora, it was postulated that there was a de-stressed zone under Lift 1 at Northparkes and under the pit at Palabora, explaining the low event rate within the column. The large events were then attributed to the stress re-adjustment around the cave, as a result of the crown pillar failing rapidly. However, in the light of the centrifuge observations, could the large events be explained by the formation of shear cracks extending from the bottom of the overlying cave, which could be the predominant mechanism of the connection itself?

Given the early stage of the research project, further work is necessary to confirm the validity of the mechanism and to rule out the possibility that is an artefact of the testing methodology. It is possible that the failure initiated at the corners of the existing modelled cave are at least in part due to interface between the sample material and the wooden spacing blocks, which lie just above the zone of failure. Another possibility is that the location of the failure zones are related to the movement of the outermost actuators, as they are approximately in line with the outermost edge of the actuators.

The observed mechanism of failure is, however, potentially consistent with analysis of seismicity found in literature for in these situations. Potvin \& Hudyma (2008) found that at Ridgeway, Palabora and Northparkes E26 Lift 2 'the rate of migration of the stress front accelerated through the de-stressed crown pillars'. They also noted that all three cases experienced large seismic events outside of the cave column, despite an overall decrease in the rate of seismicity. This was particularly evident in Northparkes E26 Lift 2, where 21 large events (local magnitude 1.0 to 2.9) were experienced in just a 13 day period (Figure 8) and the stress front migrated upwards at almost 4 metres per day (Figure 7) (Hudyma et al. 2007b). These results suggest a mechanism of cave connection which occurs very quickly, requires little energy and involves large scale sudden displacements - all of which are consistent with what is seen in the physical models.

\section{$5 \quad$ Further work}

The results shown in this paper are preliminary results and more detailed analysis of the data from the tests is currently in progress. Future research will focus on:

- Determining whether the cave connection mechanism observed (failure at the corners of the existing cave before the propagating cave breaks through vertically). Of particular interest would be to determine whether the cracks are a result of a shear mechanism which would reinforce the theory that the large seismic events after connections are associated with the formation of new major discontinuities (Ortlepp Shears). This may require further tests with an altered test setup.

- Attempting to improve the test setup to reduce settlement of the entire sample mass

- Analysing the photos using Particle Image Velocimetry (Stanier et al. 2016) to quantify displacement of the sample throughout the test and better understand the failure mechanisms.

- Processing and analysis of the acoustic emission results to understand the acoustic signature of the breakthrough.

- More tests, with the simulated cave material changed to broken pieces of the sample material instead of sand.

There is also significant potential to test different numerical modelling techniques and constitutive models, when applied to cave breakthrough. Being a controlled environment, the physical models have well understood inputs and results (in contrast to actual caving mines). Thus, the physical models can be re-created numerically and the results evaluated against the results observed in the physical models. This would give the industry a better understanding of how well current numerical models can simulate cave breakthrough. 


\section{$6 \quad$ Closing remarks}

The project involving physical modelling of a cave connection is at a very early stage, but has already provided interesting results, which prompt the questioning of previous interpretations of the mechanism involved during cave connection. In particular, the results may provide new insights on the intense period of seismic response prior to cave connection, as well as the rapid connection once the cave has propagated beyond half to three quarters of the block height. For example, it is possible that the rapid connection between upper and lower caves may not be due to a de-stressed crown pillar, as suggested in previous publications, but instead the result of the formation of new shear fractures in the periphery of the cave followed by a plug-like failure.

There is currently only four tests completed and the analysis of these tests remain very preliminary, therefore it would be premature to suggest any solid conclusions. This paper only indicates that there is the potential for unrecognised mechanisms involved in the connection of cave columns.

\section{Acknowledgement}

The authors would like to acknowledge Oz Minerals for sponsoring this research, along with Tiaan Bosman and Nicholas Schoeman for their work on the project.

\section{References}

Cuello, D \& Newcombe, G 2018, 'Key geotechnical knowledge and practical mine planning guidelines in deep, high-stress, hard rock conditions for block and panel cave mining', in Y Potvin \& J Jakubec (eds), Fourth International Symposium on Block and Sublevel Caving, 2018, Vancouver, Australian Centre for Geomechanics, pp. 17-36.

Cumming-Potvin, D 2018, 'An Extended Conceptual Model of Caving Mechanics'. PhD, The University of Western Australia.

Cumming-Potvin, D, Wesseloo, J, Jacobsz, SW \& Kearsley, EP 2018, 'A re-evaluation of the conceptual model of caving mechanics', in Y Potvin \& J Jakubec (eds), Fourth International Symposium on Block and Sublevel Caving, 2018, Vancouver, Australian Centre for Geomechanics, pp. 179-190.

Cumming-Potvin, D, Wesseloo, J, Jacobsz, SW \& Kearsley, EP 2016a, 'Fracture Banding in Caving Mines', The Journal of the Southern African Institute of Mining and Metallurgy, vol. 118, no. 8, pp. 753-761.

Cumming-Potvin, D, Wesseloo, J, Jacobsz, SW \& Kearsley, EP 2016b, 'Results from Physical Models of Block Caving', MassMin2016, 9 - 11 May 2016 2016b, Sydney, Australia, The AusIMM, pp. 329-340.

Duplancic, P \& Brady, BH 1999, 'Characterisation of Caving Mechanisms By Analysis of Seismicity And Rock Stress', 9th ISRM Congress, 25-28 August 1999 1999, Paris, France, International Society for Rock Mechanics, pp. 1049-1053.

Glazer, S 2018, Mine Seismology: Seismic Response to the Caving Process, Springer International.

Glazer, S \& Hepworth, N 2006, 'Crown pillar failure mechanism - case study based on seismic data from Palabora Mine', Mining Technology, vol. 115, no. 2, pp. 76-84.

Glazer, S \& Hepworth, N 2005, 'Seismicity Induced by Cave Mining, Palabora Experience', in Y Potvin \& M Hudyma (eds), 6th International Symposium on Rockbursts and Seismicity in Mines, 2005, Perth, Australian Centre for Geomechanics, pp. 275-280.

Hoek, E 1965, 'The Design of a Centrifuge for the Simulation of Gravitational Force Fields in Mine Models', Journal of the South African Institute of Mining and Metallurgy, vol. 65, no. 9, pp. 455-487.

Hudyma, M, Potvin, Y \& Allison, D 2007a, 'Seismic monitoring of the Northparkes lift 2 block cave_Part 1 undercutting', 1st International Symposium on Block and Sublevel Caving, 2007a, Cape Town, SAIMM, pp. 303-334.

Hudyma, M, Potvin, Y \& Allison, D 2007b, 'Seismic monitoring of the Northparkes lift 2 block cave_Part 2 production caving', 1st International Symposium on Block and Sublevel Caving, 2007b, Cape Town, SAIMM, pp. 335-354. 
Jacobsz, SW, Kearsley, EP \& Kock, JHL 2014, 'The geotechnical centrifuge facility at the University of Pretoria', in C Gaudin \& DJ White (eds), 8th International Conference on Physical Modelling in Geotechnics, 2014, Perth, Australia, Taylor \& Francis Group, pp. 169-174.

Potvin, Y \& Hudyma, M 2008, 'Interpreting caving mechanisms using microseismic monitoring data', in H Schunnesson \& E Nordlund (eds), Proceedings of MassMin 2008, Luleå, Sweden, Luleå University of Technology, pp. 971-982.

Srikant, A, Brannon, C, Flint, DC \& Casten, T 2007, 'Geotechnical characterization and design for the transition from the Grasberg open pit to the Grasberg block cave mine', 1st Canada-US Rock Mechanics Symposium, 2007, Vancouver, American Rock Mechanics Association.

Stanier, SA, Blaber, J, Take, WA \& White, D 2016, 'Improved image-based deformation measurement for geotechnical applications', Canadian Geotechnical Journal, vol. 53, no. 5, pp. 727-739.

Wesseloo, J, Cumming-Potvin, D, Potvin, Y, Jacobsz, SW, Bosman, T \& Schoeman, N 2020, 'Towards data rich verification and validation of numerical modelling predictions to cave mechanics problems', Proceedings of MassMin 2020, Santiago, Chile. 